\title{
Sex differences in behavioral responding and dopamine release during early Pavlovian learning
}

Merridee J. Lefner ${ }^{1}$, Mariana I. Dejeux ${ }^{1}$, and Matthew J. Wanat ${ }^{1}$

${ }^{1}$ Neurosciences Institute and Department of Biology, University of Texas at San Antonio, San Antonio, TX, USA.

\section{Corresponding Author:}

Matthew J. Wanat

Neurosciences Institute

Department of Neuroscience, Developmental and Regenerative Biology

University of Texas at San Antonio

One UTSA Circle

San Antonio, TX 78249

Matthew.wanat@utsa.edu

210.458 .6684

\section{Acknowledgements:}

This work was supported by National Institutes of Health grants DA033386 (MJW) and DA042362 (MJW). The authors declare no conflict of interest.

\section{Author Contributions:}

MJL and MID performed the experiments and analyzed the data. MJL and MJW designed the experiments and wrote the manuscript. 


\begin{abstract}
:
Learning associations between cues and rewards requires the mesolimbic dopamine system. The dopamine response to cues signals differences in reward value in well-trained animals. These value-related dopamine responses are absent during early learning when cues signal differences in the reward rate, which suggests cue-evoked dopamine release conveys differences between outcomes only after extensive training. However, it is unclear if this lack of value coding by cue-evoked dopamine release during early learning is unique to when cues signal differences in reward rate, or if this is also evident when cues signal differences in other value-related parameters such as reward size. To address this, we utilized a Pavlovian conditioning task in which one audio cue was associated with a small reward (one pellet) and another audio cue was associated with a large reward (three pellets). We performed fast-scan cyclic voltammetry to record changes in dopamine release in the nucleus accumbens of male and female rats throughout early learning. Cue-evoked dopamine release did not encode differences in reward value, and there were no differences in this response between males and females. However, female rats exhibited higher levels of conditioned responding and a faster latency to respond. Reward-evoked dopamine release scaled with reward size in both sexes, though there were transient sex differences in the dynamics of this response. We additionally identified sex differences in the number of post-reward head entries. Collectively these data illustrate sustained sex differences in behavioral responding as well as transient sex differences in reward-evoked dopamine release.
\end{abstract}

\title{
Introduction:
}

Efficient reward seeking involves identifying cues that predict rewards and discriminating between cues that signal different reward options. The mesolimbic dopamine system plays an integral role in regulating behavioral responses towards reward-associated cues (Phillips et al., 2007; Salamone and Correa, 2012). Cue-evoked dopamine responses convey reward-related information such as the relative reward size (Tobler et al., 2005; Roesch et al., 2007; Gan et al., 2010), reward probability (Fiorillo et al., 2003; Hart et al., 2015), and reward rate (Fonzi et al., 2017). While this effect is prominent in extensively trained animals, the 
emergence of these signals during early learning has not been well-characterized. We recently utilized a Pavlovian conditioning task to demonstrate that cue-evoked dopamine release encodes reward rate after extensive training (Fonzi et al., 2017), but not during early learning (Stelly et al., 2021). These findings suggest cue-evoked dopamine encodes reward rate through a multistep process: by first signaling an upcoming reward independent of value during early learning, and over training conveying the relative difference in value between cues. However, it is unclear if extensive training is similarly required for cue-evoked dopamine signals to convey other valuerelated parameters, such as reward size.

The aforementioned research was primarily performed in male subjects, however increasing evidence highlights sex differences in dopamine neuron activity and release (Xiao and Becker, 1994; Walker et al., 2000; McArthur et al., 2007; Becker and Chartoff, 2019; Brundage et al., 2021; Zachry et al., 2021). These differences in dopamine transmission between males and females could account for the observed sex differences in dopamine-dependent behaviors (Lynch and Carroll, 1999; Dubroqua et al., 2011; Eubig et al., 2014; Pitchers et al., 2015; Stringfield et al., 2019; Zachry et al., 2019; Kutlu et al., 2020; Chen et al., 2021; George et al., 2021). While certain forms of Pavlovian conditioned responding can differ between males and females (Stringfield et al., 2019), it is not known if sex differences during Pavlovian learning is accompanied by distinct patterns of dopamine signaling.

In this study, we trained male and female rats on a Pavlovian task where one cue was associated with a small reward (one pellet) and another cue was associated with a large reward (three pellets). We used fast-scan cyclic voltammetry to record changes in dopamine release in the nucleus accumbens (NAc) during early learning. Female rats displayed higher levels of conditioned responding, a faster latency to the food port, and a higher number of post-reward head entries compared to male rats. The cue-evoked dopamine response correlated with conditioned responding but did not encode differences in reward size during early training sessions. There were no differences in cue-evoked dopamine release between males and females. In contrast, the dynamics of reward-evoked dopamine release was influenced by both reward size and sex. These data illustrate that sex differences in dopamine transmission are evident in response to rewards but not their predictors. 


\section{Methods:}

\section{Subjects and surgery}

All procedures were approved by the Institutional Animal Care and Use Committee at the University of Texas at San Antonio. Male and female Sprague-Dawley rats (Charles River, MA) weighing 300-350 g were pair-housed upon arrival and given ad libitum access to water and chow and maintained on a 12-hour light/dark cycle. Carbon fiber voltammetry electrodes consisted of a carbon fiber housed in silica tubing and cut to a length of $150 \mu \mathrm{m}$ (Clark et al., 2010). Voltammetry electrodes were surgically implanted to target the NAc (relative to bregma: $1.3 \mathrm{~mm}$ anterior; $\pm 1.3 \mathrm{~mm}$ lateral; $7.0 \mathrm{~mm}$ ventral) along with a $\mathrm{Ag} / \mathrm{AgCl}$ reference electrode. Rats were single-housed following surgery and allowed to recover for $>3$ weeks before beginning training.

\section{Behavioral procedures}

After recovering from surgery, rats were placed and maintained on mild food restriction ( $\sim 15 \mathrm{~g}$ /day of standard lab chow) to target $90 \%$ free-feeding weight, allowing for an increase of $1.5 \%$ per week. Behavioral sessions were performed in chambers (Med Associates) that had grid floors, a house light, a food tray, and auditory stimulus generators ( 2.5 and $4.5 \mathrm{kHz}$ tones). To familiarize rats with the chamber and food retrieval, rats underwent a single magazine training session in which 20 food pellets (45 mg, BioServ) were non-contingently delivered at a $90 \pm 15 \mathrm{~s}$ variable interval. Rats then underwent six Pavlovian conditioning sessions (1/day) that each consisted of 50 trials where the termination of a $5 \mathrm{~s}$ audio cue $(\mathrm{CS} ; 2.5 \mathrm{kHz}$ tone or $4.5 \mathrm{kHz}$ tone, counterbalanced across animals) resulted in the delivery of a single food pellet (Small Reward trials) or three food pellets (Large Reward trials) and illumination of the food port light for $4.5 \mathrm{~s}$. Each session contained 25 Small Reward trials and 25 Large reward trials delivered in a pseudorandom order, with a $45 \pm 5 \mathrm{~s}$ ITI between all trials. Conditioned responding was quantified as the change in the rate of head entries during the $5 \mathrm{~s}$ CS relative to the $5 \mathrm{~s}$ preceding the CS delivery (Fonzi et al., 2017; Stelly et al., 2021). We also quantified the latency to initiate a 
head entry during the CS. For the post-US analysis, we calculated the number of head entries made during the Early (0-3 s), Mid (3-6 s), and Late (6-9 s) epochs following reward delivery.

\section{Voltammetry recordings and analysis}

Chronically-implanted electrodes were connected to a head-mounted amplifier to monitor changes in dopamine release in behaving rats using fast-scan cyclic voltammetry as described previously (Clark et al., 2010; Fonzi et al., 2017; Oliva and Wanat, 2019; Stelly et al., 2019; Stelly et al., 2020; Oliva et al., 2021; Stelly et al., 2021). The carbon fiber electrodes were held at $-0.4 \mathrm{~V}$ (vs. $\mathrm{Ag} / \mathrm{AgCl}$ ) with voltammetric scans applied at $10 \mathrm{~Hz}$ in which the potential was ramped in a triangular waveform to $+1.3 \mathrm{~V}$ and back to $-0.4 \mathrm{~V}$ at a rate of $400 \mathrm{~V} / \mathrm{s}$. Chemical verification of dopamine was achieved by obtaining a high correlation of the cyclic voltammogram during a reward-related event to that of a dopamine standard (correlation coefficient $r^{2} \geq 0.75$ by linear regression). Voltammetry data and corresponding behavioral data for a session were excluded from analysis if the detected voltammetry signal did not satisfy this chemical verification criteria (Fonzi et al., 2017; Stelly et al., 2021). Chemometric analysis (Heien et al., 2005) was performed on the voltammetry signal using a standard training set that accounts for dopamine, $\mathrm{pH}$, and drift. The dopamine concentration was estimated based on the average post-implantation sensitivity of electrodes $(34 \mathrm{nA} / \mu \mathrm{M})($ Clark et al., 2010). Individual trials were excluded if chemometric analysis failed to identify dopamine on $>25 \%$ of the data points.

The CS-evoked dopamine response was quantified as the average dopamine response during the 5 s CS relative to the 5 s prior to the CS delivery (Fonzi et al., 2017; Stelly et al., 2021). The US-evoked dopamine response was quantified as the average dopamine response during three 3 s time windows (Early: 0-3 s; Mid: 3-6 s; Late: 6-9 s) following the reward delivery, relative to the $0.5 \mathrm{~s}$ preceding the reward delivery. 


\section{Data analysis}

Statistical analyses were performed in GraphPad Prism 9 and RStudio. Data were analyzed in five trial bins or averaged within the session. Behavioral responding and dopamine quantification were analyzed using a mixed-effects model fit (restricted maximum likelihood method), repeated measures where appropriate, followed by a post hoc Sidak's test. The GeisserGreenhouse correction was applied to address unequal variances between groups. A repeated measures correlation was used to correlate dopamine signals and behavioral outcomes across sessions (Bakdash and Marusich, 2017; Stelly et al., 2021). The list of statistical analyses is presented in the Supplementary Table 1.

\section{Histology}

Rats were deeply anesthetized, and electrical lesions were applied to the voltammetry electrodes followed by intracardial perfusion with $4 \%$ paraformaldehyde. Brains were removed and postfixed for at least 24 hours, then subsequently placed in $15 \%$ and $30 \%$ sucrose solutions in phosphate-buffered saline. Brains were then flash frozen on dry ice, coronally sectioned, and stained with cresyl violet. Electrode locations were mapped onto a standardized rat brain atlas.

\section{Results:}

Rats were trained on a Pavlovian conditioning paradigm in which one audio cue (CS) signaled the delivery of a single sucrose pellet (US; Small Reward trial) and another audio cue signaled the delivery of three sucrose pellets (Large Reward trial, Fig. 1A). Conditioned responding was quantified as the change in the rate of head entries during the $5 \mathrm{~s}$ CS relative to the rate of head entries during the 5 s preceding the CS (Fonzi et al., 2017; Stelly et al., 2020; Stelly et al., 2021). Rats increased conditioned responding across sessions, with no difference between Small and Large Reward cues (three-way mixed-effects analysis; session effect: $F_{(2.26 \text {, }}$ $24.86)=14.01, p<0.0001$; reward size effect: $F_{(1,11)}=0.03, p=0.86 ; n=13$ rats, Fig. 1B). There was a trend for enhanced conditioned responding in female rats (sex effect: $F_{(1,55)}=3.90, p=$ 0.05; session $\mathrm{x}$ sex interaction: $F_{(5,55)}=2.34, p=0.05$; Fig. 1B). Rats also decreased the latency to the food port across training sessions, with no difference between Small and Large reward 
A

Small Reward trial (25 trials)

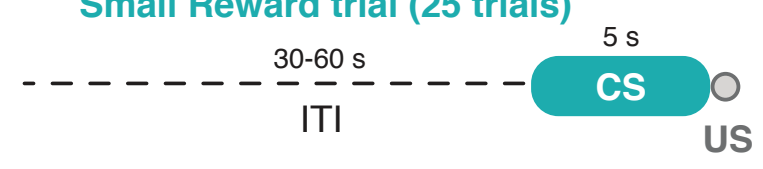

Pavlovian task (50 trials per session)

Large Reward trial (25 trials)

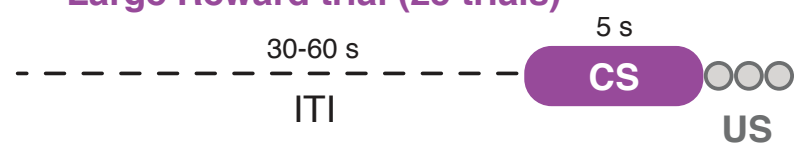

Conditioned responding

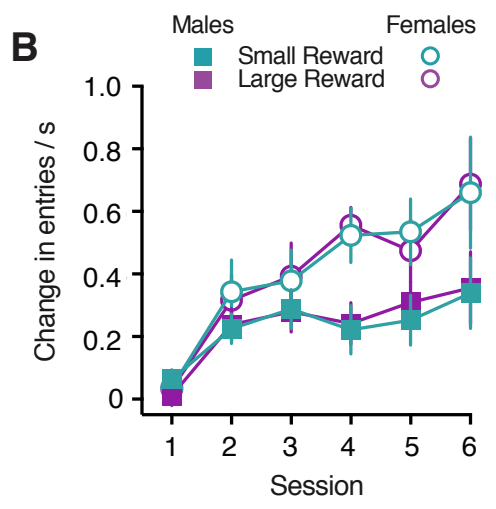

C Sessions 1-3
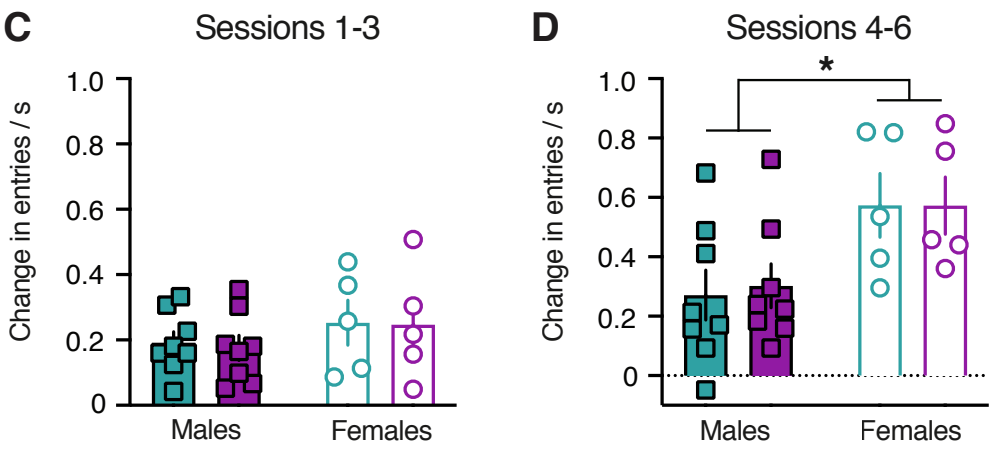

Latency to food port

E

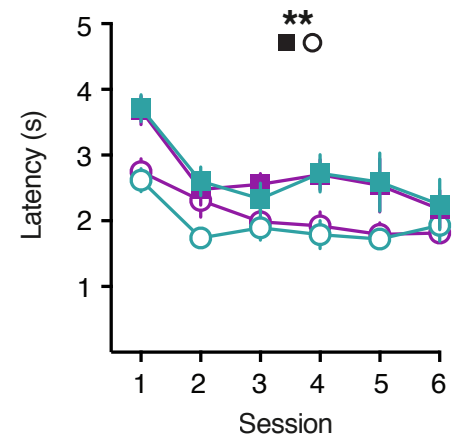

$\mathbf{F}$

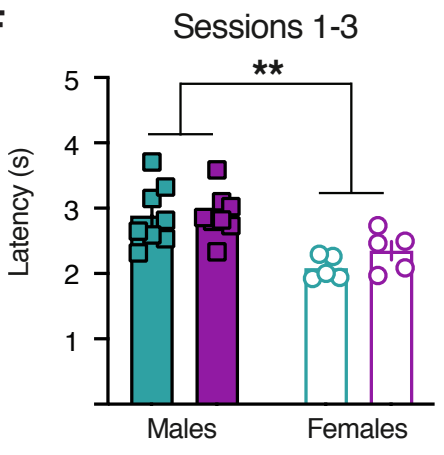

Sessions 4-6

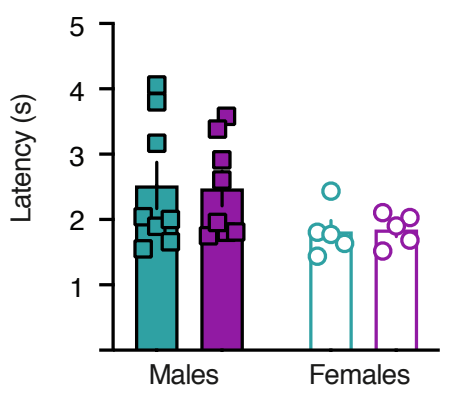

Figure 1. Sex differences in behavioral responding during CS presentation. (A) Training schematic for Pavlovian reward size task. (B) Conditioned responding for males (filled squares) and females (open circles) during Small Reward (teal) and Large Reward (purple) trials. (C) Conditioned responding averaged across the first three sessions of training. (D) Conditioned responding averaged across the latter three sessions of training. (E) Latency to respond to the food port (F) Latency to respond averaged across the first three sessions of training. (D) Latency to respond averaged across the latter three sessions of training. $* p<0.05, * * p<0.01$.

trials (three-way mixed-effects analysis; session effect: $F_{(2.94,32.33)}=6.26, p<0.002$; reward size effect: $F_{(1,11)}=0.51, p=0.49$; Fig. 1E). Females displayed a faster latency to respond across sessions compared to males (sex effect: $F_{(1,55)}=8.80, p=0.004$; Fig. 1E), consistent with prior findings (Eubig et al., 2014; Stringfield et al., 2019). We further analyzed these behavioral responses when averaged into three-session bins. During the first three sessions there were no 
sex differences in conditioned responding (two-way mixed-effects analysis; sex effect: $F_{(1,11)}=$ 0.90, $p=0.36$; Fig. 1C), though females exhibited a faster latency to enter the food port (twoway mixed-effects analysis; sex effect: $F_{(1,11)}=14.56, p=0.003$; Fig. 1F). During the latter three sessions, female rats displayed higher levels of conditioned responding (two-way mixed-effects analysis; sex effect: $F_{(1,11)}=5.11, p<0.05$; Fig. 1D), though there were no sex differences in the latency to respond (two-way mixed-effects analysis; sex effect: $F_{(1,11)}=2.71, p=0.13$; Fig. 1G). Collectively these findings illustrate sex differences in behavioral responding within the CS presentation during early Pavlovian learning. However, the behavioral responses during the cue presentation did not reflect differences in the upcoming reward size.

Given the sex differences in CS-evoked behavior, we next examined if male and female rats differed in their behavioral responses following the reward delivery. Female rats performed a higher number of non-CS head entries relative to males (Supplementary Fig. 1), which suggests females performed more head entries following the US. To address this possibility, we examined the head entries in three separate post-reward epochs (Fig. 2A). During the first $3 \mathrm{~s}$ post reward delivery (Early US), rats made a greater number of head entries following the delivery of the Large Reward which likely reflects consummatory responses (three-way mixed-effects analysis; reward size effect: $F_{(1,11)}=22.95, p=0.0006$; Fig. 2B). During the first three sessions, female rats performed more head entries following the Large Reward delivery compared to Small Reward trials and compared to male rats (two-way mixed-effects analysis; reward size effect: $F_{(1}$, 11) $=14.96, p=0.003$; post hoc Sidak's test - Reward size; Females: $t_{(11)}=3.76, p=0.006$; post hoc Sidak's test - Sex; Large Reward: $t_{(22)}=2.43, p<0.05$; Fig. 2C). Females continued to show increased head entries in Large Reward trials during the last three sessions (two-way mixedeffects analysis; reward size effect: $F_{(1,11)}=16.48, p=0.002$; post hoc Sidak's test - Reward size; Females: $t_{(11)}=3.82, p=0.006$; Fig. 2D).

Whereas rats performed a greater number of head entries following the Large Reward delivery during the Early US period, rats performed more head entries following the Small Reward delivery during the 3-6 s post reward period (Mid US) (three-way mixed-effects analysis; reward size effect: $F_{(1,11)}=7.52, p=0.02$; Fig. 2E). Females demonstrated a greater number of head entries compared to males throughout the Mid US period (sex effect: $F_{(1,55)}=$ 
A
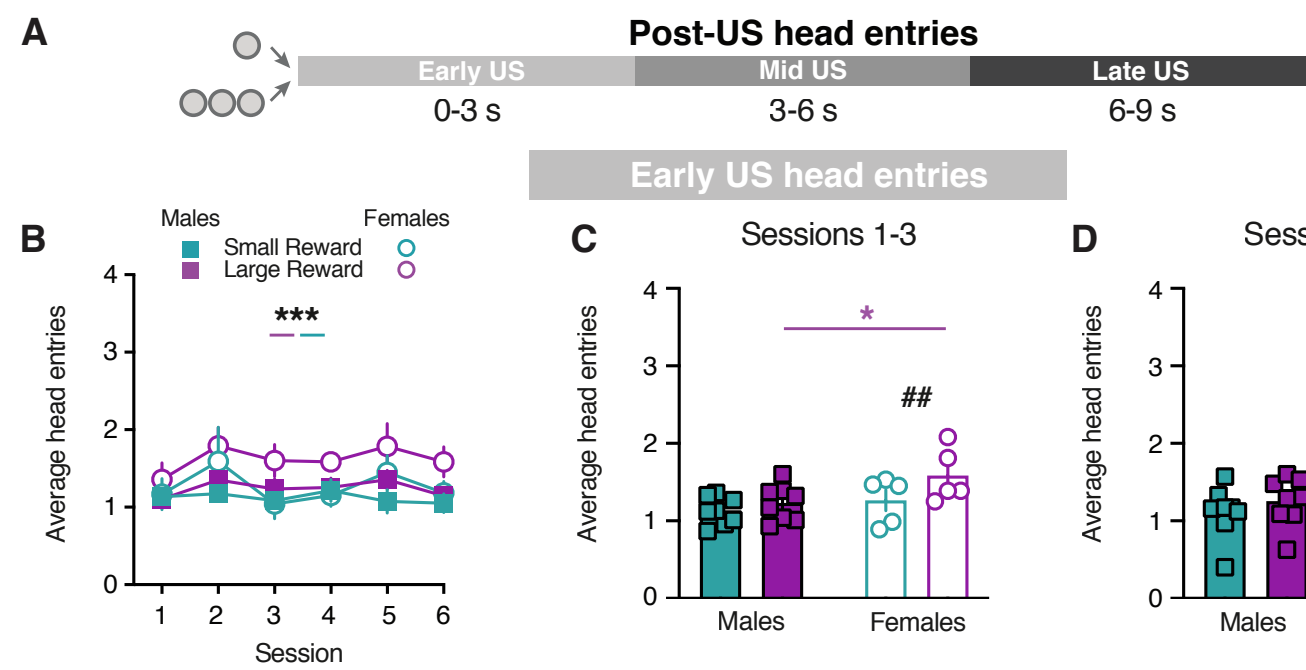

$\begin{array}{lll} & \text { Early US head entries } \\ \text { C } & \text { Sessions } 1-3 \quad \text { D }\end{array}$

Sessions 4-6
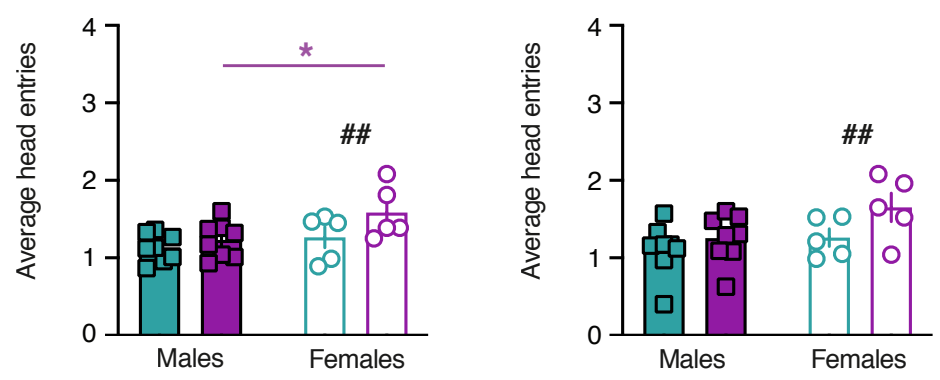

\section{Mid US head entries}

E

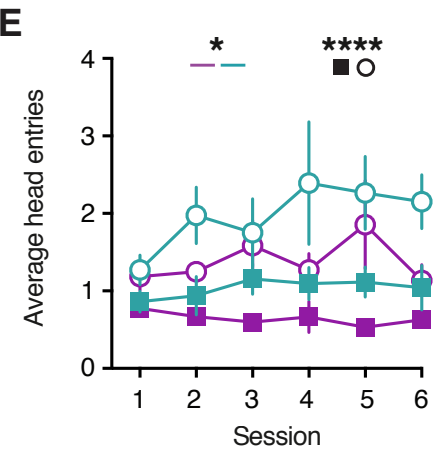

$\mathbf{F}$

Sessions 1-3
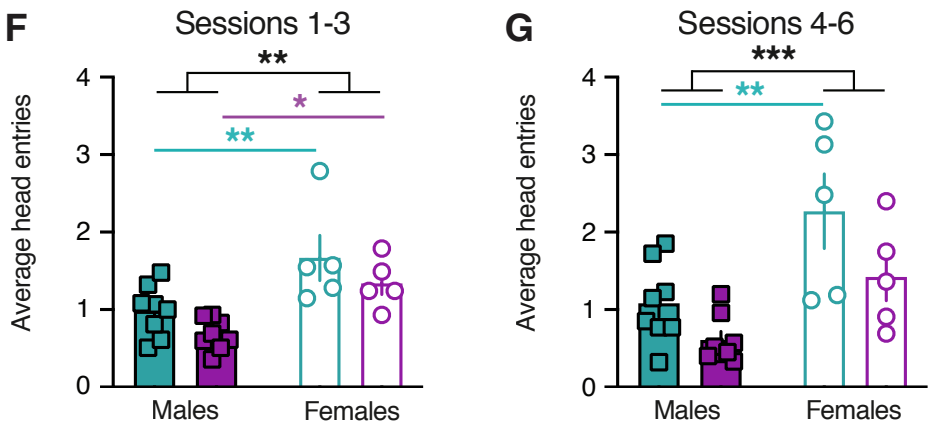

Late US head entries

H

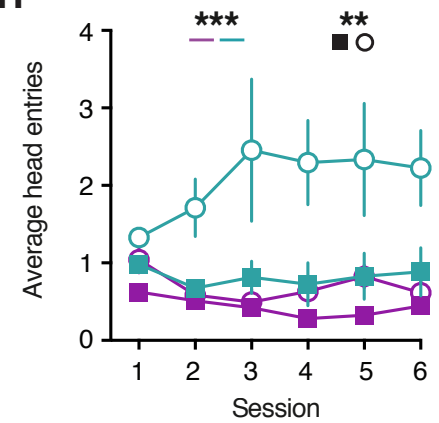

I

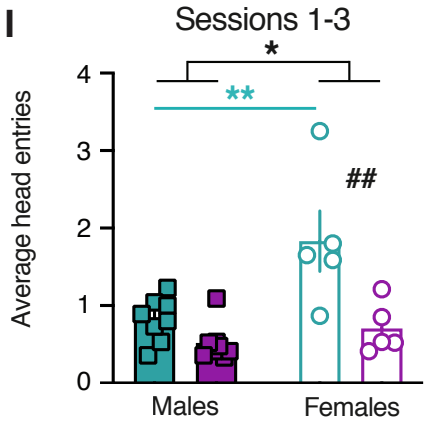

$\mathbf{J}$

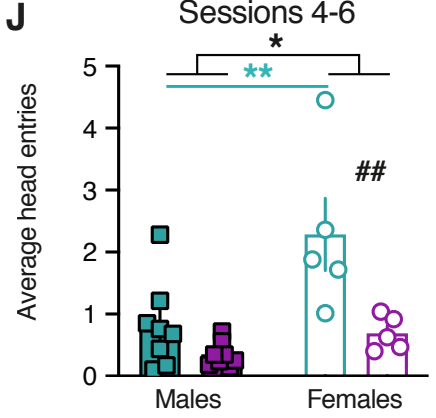

Figure 2. Sex differences in behavioral responding during US presentation. (A) Schematic for post-US epochs: Early US (0-3 s), Mid US (3-6 s), and Late US (6-9 s). (B) Average head entries made during the Early US for Small Reward and Large Reward trials. (C) Early US head entries averaged across the first three sessions of training. (D) Early US head entries averaged across the latter three sessions of training. (E) Average head entries made during the Mid US for Small Reward and Large Reward trials. (F) Mid US head entries averaged across the first three sessions of training. (G) Mid US head entries averaged across the latter three sessions of training. (H) Average head entries made during the Late US for Small Reward and Large Reward trials. (I) Late US head entries averaged across the first three sessions of training. (J) Late US head entries averaged across the latter three sessions of training. * denotes main effect of sex or post-hoc effect of sex, ${ }^{*} p<0.05, * * p<0.01,{ }^{*} * * p<0.001, * * * * p<$ 0.0001. \# denotes post-hoc effect of reward size, \#\# $p<0.01$. 
19.40, $p<0.0001$; Fig. 2E). The influence of sex and reward size on head entries was evident during both the first three sessions (two-way mixed-effects analysis; reward size effect: $F_{(1,11)}=$ 8.83, $p=0.01$; sex effect: $F_{(1,11)}=12.65, p=0.005$; post hoc Sidak's test - Sex; Small Reward: $t_{(22)}=3.15, p=0.009$; Large Reward: $t_{(22)}=3.04, p=0.01$; Fig. 2F) and the last three sessions (two-way mixed-effects analysis; reward size effect: $F_{(1,22)}=6.59, p=0.02$; sex effect: $F_{(1,22)}=$ 15.04, $p=0.0008$; post hoc Sidak's test - Sex; Small Reward: $t_{(22)}=3.25, p=0.007$; Fig. 2G).

In the 6-9 s post reward period (Late US) there was an interaction between sex and reward size, with female rats exhibiting a higher number of head entries following Small Reward trials (three-way mixed-effects analysis; sex x reward size effect: $F_{(1,55)}=6.77, p=0.01$; sex effect: $F_{(1,55)}=9.74, p=0.003$; reward size effect: $F_{(1,11)}=21.63, p=0.0007$; Fig. 2H). The effects of reward size and sex on the number of head entries was evident during the first three sessions (two-way mixed-effects analysis; reward size effect: $F_{(1,11)}=23.81, p=0.0005$; sex effect: $F_{(1,11)}=8.12, p=0.02$; reward size x sex effect: $F_{(1,11)}=7.83, p=0.02$; post hoc Sidak's test - Sex; Small Reward: $t_{(22)}=3.94, p=0.001$; post hoc Sidak's test - Reward size; Females: $t_{(11)}=4.89, p=0.001$; Fig. 2I) and last three sessions of training (two-way mixed-effects analysis; reward size effect: $F_{(1,11)}=14.53, p=0.003$; sex effect: $F_{(1,11)}=9.52, p=0.01$; post hoc Sidak's test - Sex; Small Reward: $t_{(22)}=3.69, p=0.003$; post hoc Sidak's test - Reward size; Females: $t_{(11)}=3.77, p=0.006$; Fig. 2J). These results demonstrate that both sex and reward size influence the number of post reward head entries during early Pavlovian learning.

The emergence of Pavlovian conditioned responses depends upon dopamine signaling within the ventral striatum (Darvas et al., 2014). Here, we performed voltammetry recordings in the NAc to examine how the CS- and US-evoked dopamine responses progressed across training (males: $n=9$ electrodes; females: $n=5$ electrodes; Fig. 3A-B). Both male and female subjects exhibited increases in dopamine release to the CS presentation (Fig. 3C). We quantified CSevoked dopamine release as the average response during the $5 \mathrm{~s}$ CS relative to the $5 \mathrm{~s}$ prior to the CS, identical to the manner in which conditioned responding was calculated (Fig. 1). CS-evoked dopamine release did not differ between sexes or trial type (three-way mixed-effects analysis; session effect: $F_{(1.97,23.64)}=3.22, p=0.06$; sex effect: $F_{(1,30)}=0.07, p=0.80$; reward size effect: $F_{(1,12)}=3.54, p=0.09$; Fig. 3D). 
A

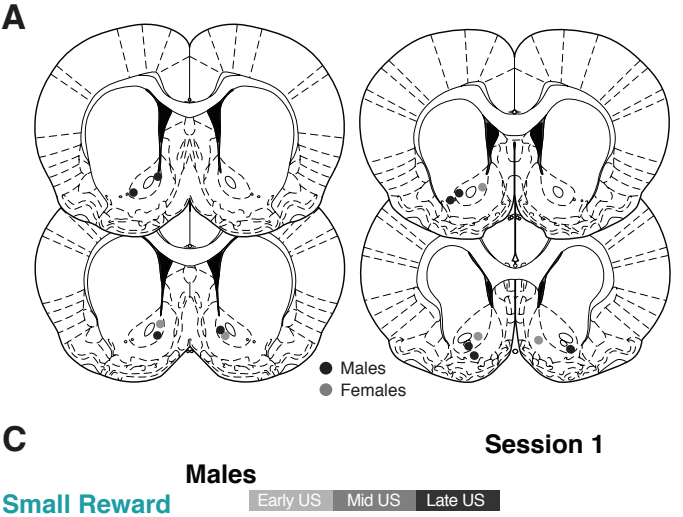

B

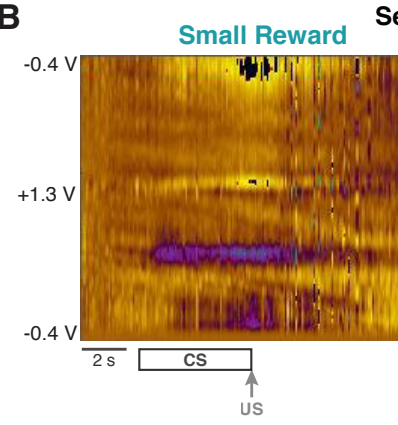

Session 3

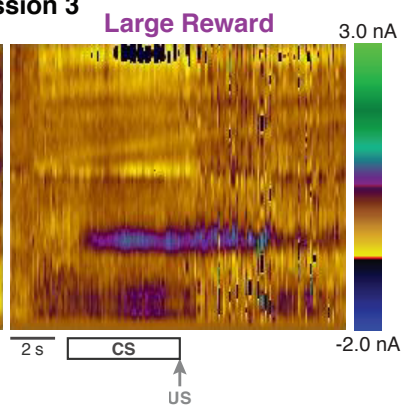

Females

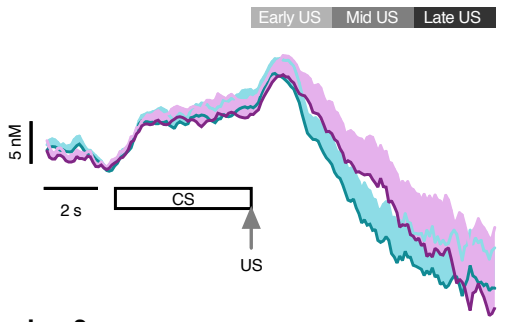

Large Reward

Session 3
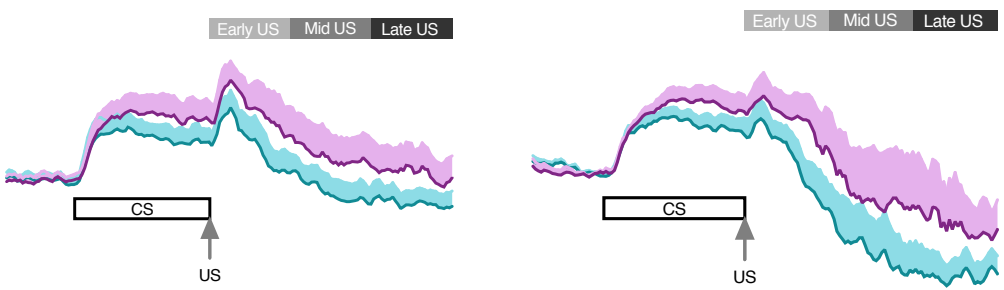

Session 4
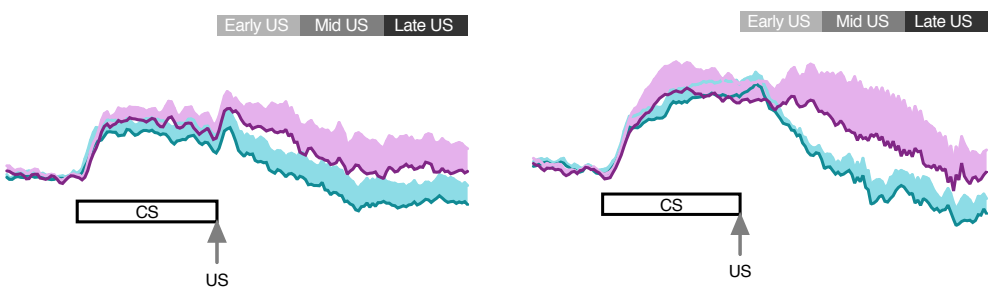

Session 6
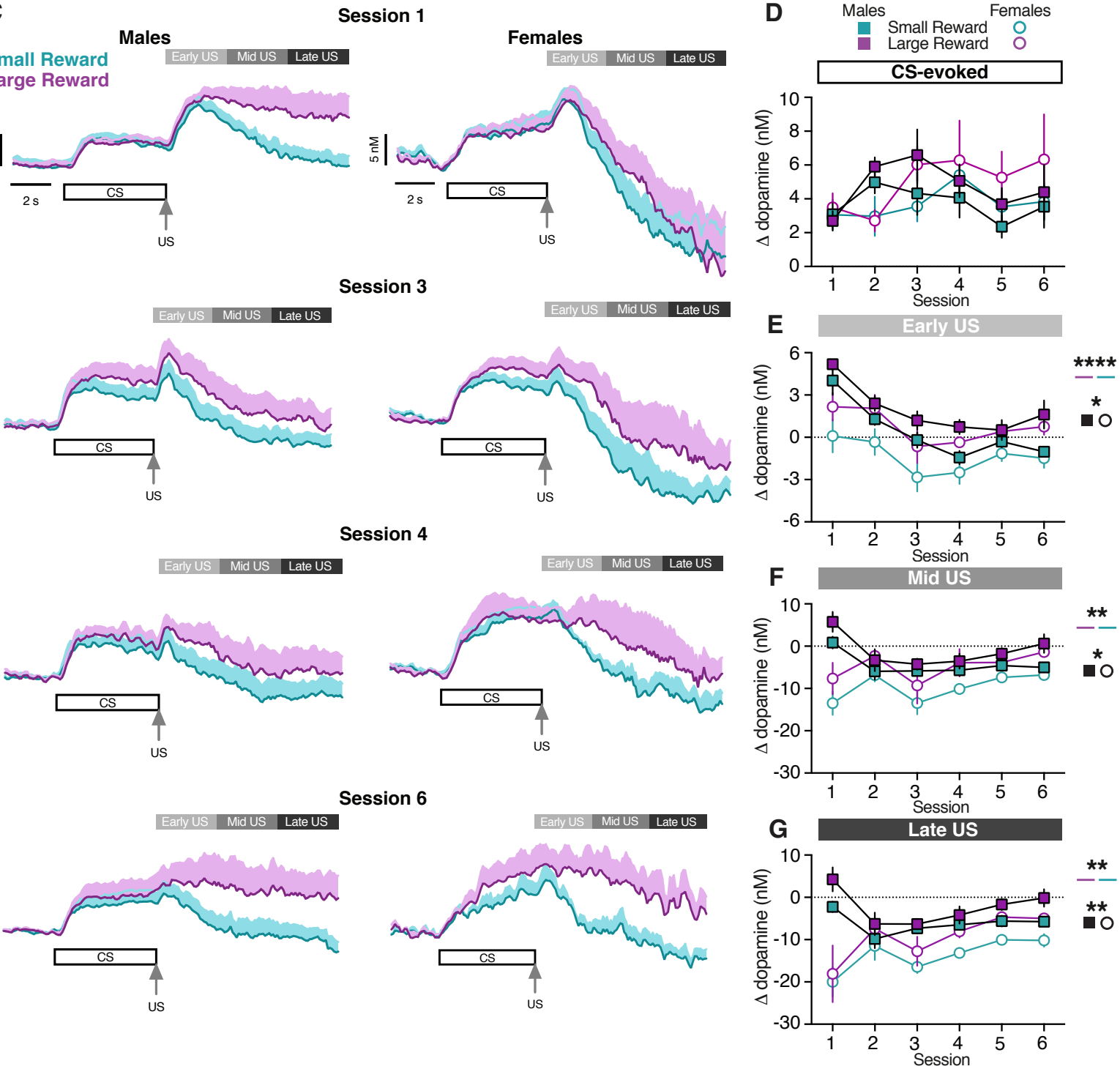

Figure 3. Dopamine release in the NAc during early training sessions. (A) Location of voltammetry electrodes in males (black) and females (gray). (B) Representative two-dimensional pseudocolor plots of the resulting current from voltage sweeps (y axis) as a function of time (x axis) of voltammetry recordings in the NAc. (C) Average dopamine signals across training sessions in males (left) and females (right). (D) Average CS-evoked dopamine release across sessions. (E) Average Early USevoked dopamine release across sessions. (F) Average Mid US-evoked dopamine release across sessions. (G) Average Late US-evoked dopamine release across sessions. $* p<0.05, * * p<0.01, * * * *$ $p<0.0001$. 
Male and female rats displayed increased dopamine release in response to the delivery of both the Small and Large Rewards (Fig. 3C). In contrast to the elevated post-reward dopamine response in males, female reward-evoked dopamine release was followed by a prolonged drop during the first session (Fig. 3C). To quantify the differences observed in the dopamine dynamics following US delivery, we averaged the dopamine response during the Early (0-3 s), Mid (3-6 s), and Late (6-9 s) US epochs. The Early US-evoked dopamine response was elevated during Large Reward trials, but remained lower in females compared to males across training (three-way mixed-effects analysis; sex effect: $F_{(1,30)}=5.17, p=0.03$; reward size effect: $F_{(1,12)}=39.44, p<$ 0.0001; Fig. 3E). There was a sex x session interaction during the Mid US-evoked dopamine response (three-way mixed-effects analysis; session effect: $F_{(2.29,27.50)}=2.95, p=0.06$; sex effect: $F_{(1,30)}=5.01, p=0.03$; reward size effect: $F_{(1,12)}=15.62, p=0.002$; session x sex effect: $F_{(5,30)}=5.31, p=0.001$; Fig. 3F). Similarly, there was a sex x session interaction during the Late US-evoked dopamine response (three-way mixed-effects analysis; session effect: $F_{(1.70,20.42)}=$ 2.04, $p=0.16$; sex effect: $F_{(1,30)}=10.61, p=0.003$; reward size effect: $F_{(1,12)}=13.47, p=0.003$; session x sex effect: $F_{(5,30)}=6.60, p=0.0003$; Fig. 3G). These results suggest that in contrast to cue-evoked dopamine release, reward-evoked dopamine release encodes differences in reward size throughout the post-reward period. Additionally, female rats exhibited a smaller rewardevoked dopamine response relative to male rats.

The most notable sex difference in the US-evoked dopamine response was during the first training session. When the first session was removed from analyses there was no longer an interaction between sex and session during the Mid US period (session x sex effect: $F_{(4,24)}=$ $1.46, p=0.24)$ or the Late US period (session $\mathrm{x}$ sex effect: $F_{(4,24)}=1.58, p=0.21$ ). We next performed a within-session analysis on the first two sessions to determine when the transient Mid and Late US-evoked dopamine dip in females attenuated. The Mid US-evoked dopamine response was lower in females during the first session (three-way mixed-effects analysis Session 1; sex effect: $F_{(1,30)}=15.45, p=0.0005$; Fig. 4A), but this was no longer observed by the second session (three-way mixed-effects analysis - Session 2; sex effect: $F_{(1,33)}=0.05, p=$ 0.83; Fig. 4A). The same phenomenon was observed for the Late US-evoked dopamine release in females between the first and second session (three-way mixed-effects analysis - Session 1; 

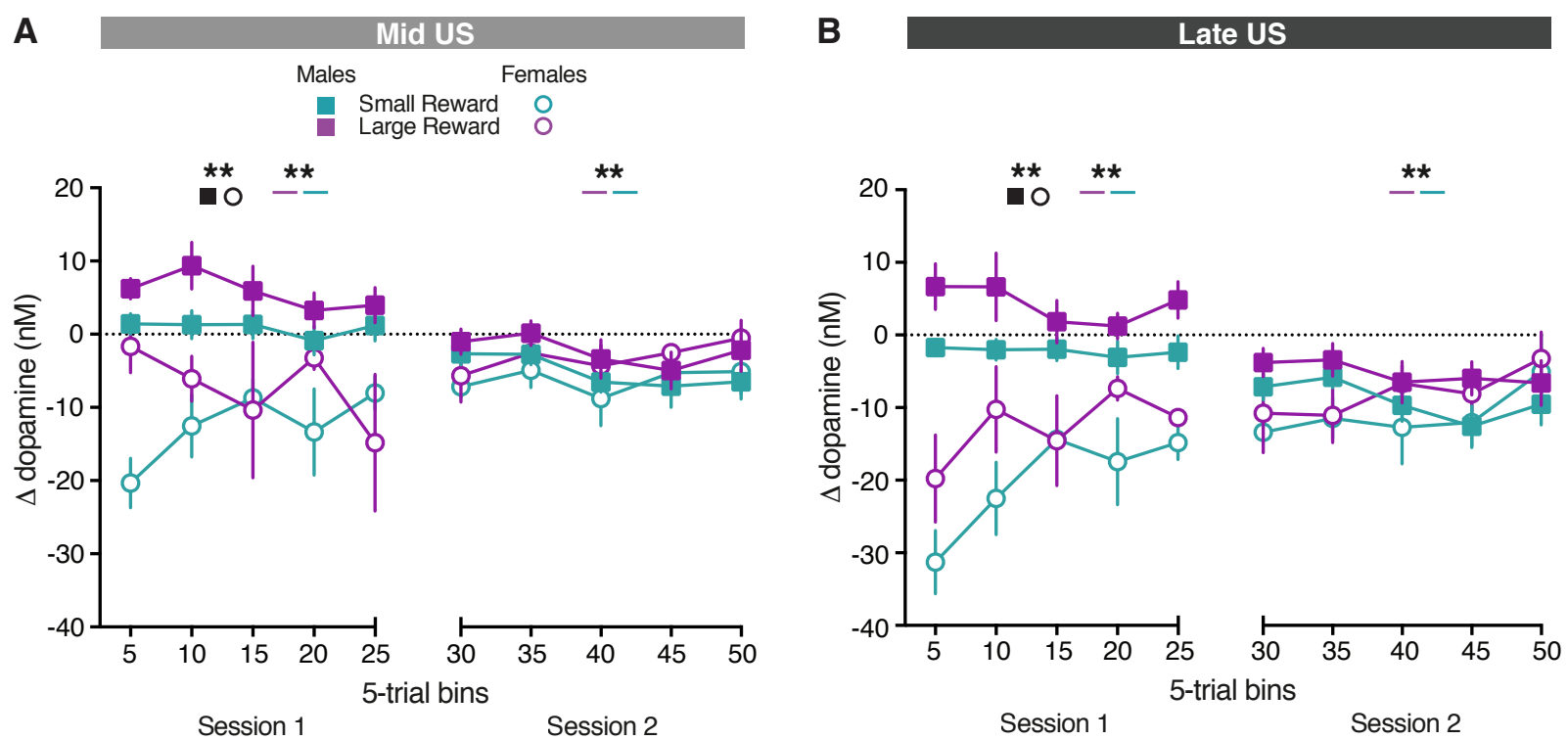

Figure 4. Average Mid and Late US-evoked dopamine response during first two training sessions. (A) Average Mid US-evoked dopamine response in five trial bins during first two training sessions. (B) Average Late US-evoked dopamine response in five trial bins during first two training sessions. $*_{*}^{*} p<$ 0.01 .

sex effect: $F_{(1,24)}=27.73, p<0.0001$; Session 2; sex effect: $F_{(1,34)}=0.26, \mathrm{p}=0.61$; Fig. 4B). These findings suggest that the sex differences observed in the Mid and Late US-evoked dopamine response are constrained to the first session.

We utilized a repeated measures correlation analysis to determine if behavioral responding relates to dopamine release during the CS or following the US delivery. There was no difference between Small and Large trials on conditioned responding, the latency to the food port, or CS-evoked dopamine release (Figs. 1, 3). As such, we combined the data between trial types for analyses during the CS. Conditioned responding was positively correlated with CSevoked dopamine release in both male and female rats (repeated measures correlation; males: $\mathrm{r}_{\mathrm{rm}}$ $=0.13, p=0.006$; females: $r_{r m}=0.53, p=0.02$; Fig. 5A). In contrast, the latency to the food port was not related to CS-evoked dopamine release (repeated measures correlation; males: $\mathrm{r}_{\mathrm{rm}}=$ $-0.05, p=0.33$; females: $r_{\mathrm{rm}}=-0.07, p=0.26$; Fig. 5B). Correlating the post-reward head entries to dopamine release during the equivalent Early, Mid, and Late time epochs did not identify any relationship between head entries and US-evoked dopamine. We next examined if post-reward head entries were related to dopamine release during the entire $9 \mathrm{~s}$ post-US epoch (Supplementary Fig. 2). Across the post-reward period, head entries were not related to 
A

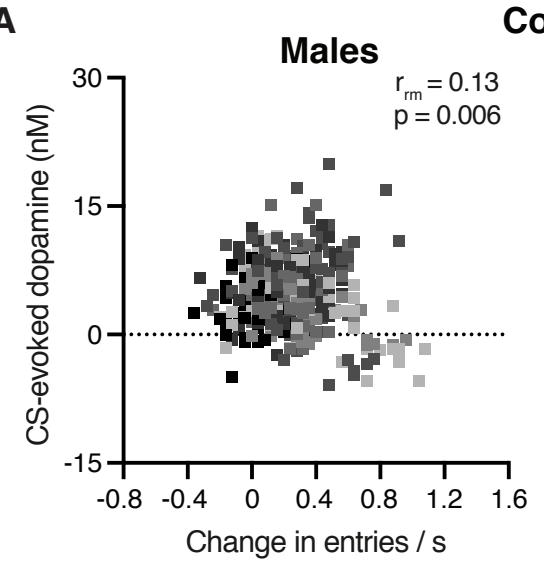

B

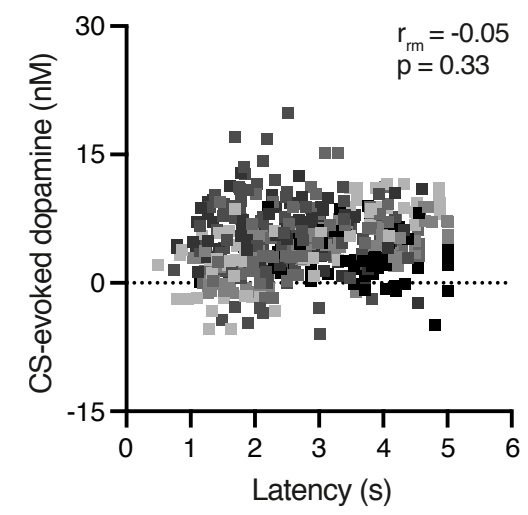

Conditioned responding

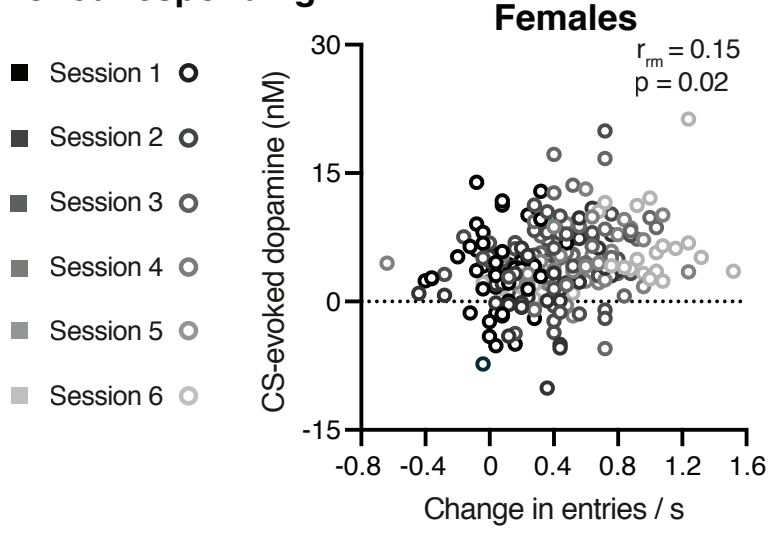

Latency to food port
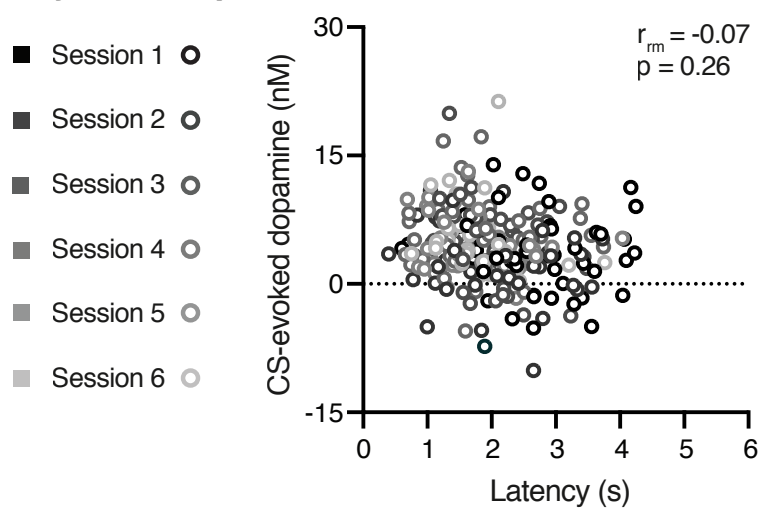

C

Post US head entries: Small Reward

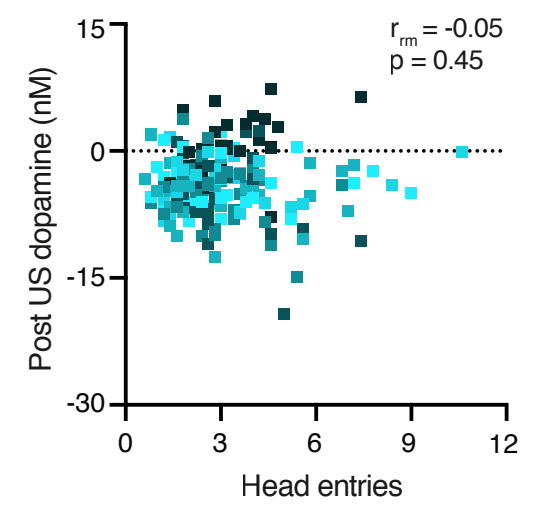

- Session 10
- Session 20
- Session 30
Session 40
Session 50

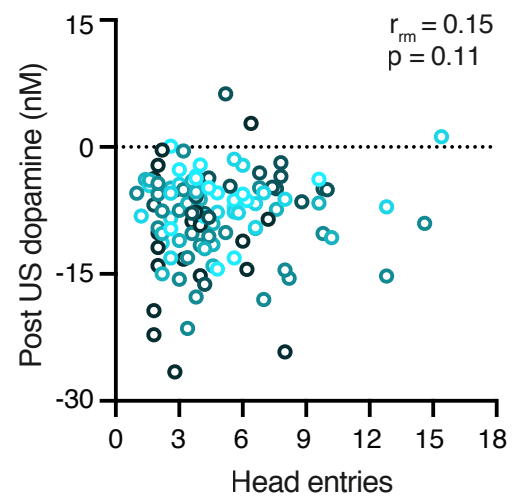

D

Post US head entries: Large Reward

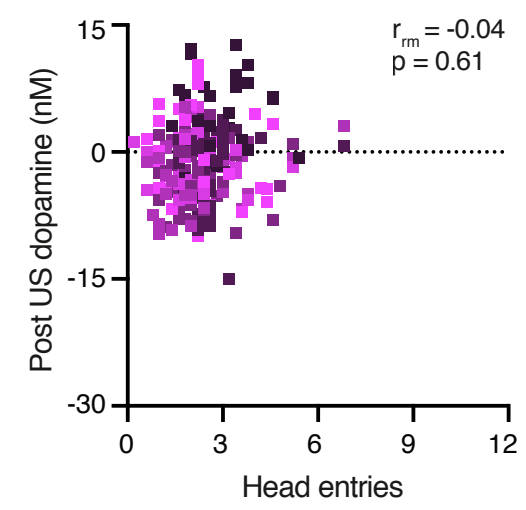

- Session 10

- Session 20

- Session 30

- Session 4 o

- Session 50

- Session 6

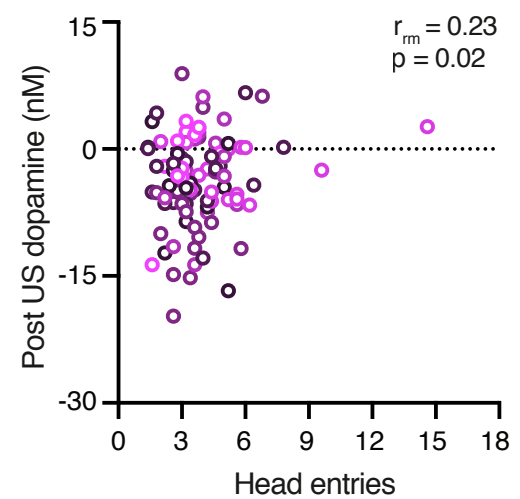


Figure 5. Relationship between CS and US-evoked dopamine and behavioral responding. (A) Relationship between CS-evoked dopamine release and conditioned responding in males (left) and females (right). (B) Relationship between CS-evoked dopamine release and the latency to the food port. (C) Relationship between post US-evoked dopamine release and the number of post-US head during Small Reward trials. (D) Relationship between post US-evoked dopamine release and the number of post-US head entries during Large Reward trials.

dopamine release during Small Reward trials (repeated measures correlation; males: $r_{\mathrm{rm}}=-0.05$, $p=0.45$; females: $\mathrm{r}_{\mathrm{rm}}=0.15, p=0.11$; Fig. 5C). However, there was a positive relationship between head entries and post-US dopamine release during Large Reward trials in female rats (repeated measures correlation; males: $\mathrm{r}_{\mathrm{rm}}=-0.04, p=0.61$; females: $\mathrm{r}_{\mathrm{rm}}=0.23, p=0.02$; Fig. 5D). Collectively, our results identified sex differences in both behavioral responding and dopamine dynamics during early Pavlovian learning.

\section{Discussion:}

The dopamine response to cues can signal differences in value-related information in well-trained animals (Fiorillo et al., 2003; Tobler et al., 2005; Roesch et al., 2007; Gan et al., 2010; Hart et al., 2015; Fonzi et al., 2017). For example, cue-evoked dopamine release conveys differences in the reward rate after extensive training (Fonzi et al., 2017). However, cue-evoked dopamine release does not signal differences in reward rate during early Pavlovian training sessions (Stelly et al., 2021). Our current results extend on these findings and demonstrate that cue-evoked dopamine release does not signal differences in reward size during early training sessions. Together this suggests that cue-evoked dopamine signals differences in reward value through a multistep process. During early training cue-evoked dopamine signals an upcoming reward independent of value. Additional training is then required for cue-evoked dopamine release to encode differences in reward value.

Prior studies have identified sex differences in a variety of dopamine-dependent behaviors (Zachry et al., 2019; Kutlu et al., 2020; Chen et al., 2021). Female subjects display a faster acquisition rate and elevated responding during drug self-administration (Lynch and Carroll, 1999; George et al., 2021). Females exhibit higher levels of conditioned freezing relative to males during fear conditioning (Dubroqua et al., 2011). Sex differences have also been identified in Pavlovian conditioning tasks employing food rewards (Stringfield et al., 2019). 
Specifically, female rats exhibited greater levels of sign-tracking behavior (e.g., physical interactions with a lever cue) but there were no sex differences in goal-tracking behavior (e.g., head entries to the food receptacle)(Stringfield et al., 2019). In contrast, we found that females exhibited a higher level of goal-tracking behavior in our Pavlovian paradigm using audio cues. Collectively these studies illustrate that sex differences in reward learning may depend upon the type of cue (e.g., audio vs. lever extension) and the form of conditioned responding expressed by the subject.

The behavioral analyses in Pavlovian conditioned tasks traditionally focuses on the behavioral responses during the cue. Here, we additionally analyzed the post-reward head entries into the food port. Both males and females performed more head entries immediately following the delivery of the large reward option. After these initial consummatory responses, female rats made more head entries following small reward trials relative to large reward trials. These unreinforced head entries to the food port suggest females were expecting a large reward on small reward trials. These findings illustrate previously unappreciated sex differences in behavioral responding following the delivery of rewards.

Prior studies demonstrate that females have more dopamine neurons in the ventral tegmental area (McArthur et al., 2007; Zachry et al., 2021). Furthermore, estrous cycle hormones are capable of influencing the firing rate of dopamine neurons in the ventral tegmental area and modulating dopamine release in the striatum (Castner et al., 1993; Xiao and Becker, 1994; Zhang et al., 2008; Becker et al., 2012; Calipari et al., 2017; Becker and Chartoff, 2019; Yoest et al., 2019; Zachry et al., 2021). The sex differences in dopamine transmission can result from intrinsic differences between males and females as well as estrous cycle hormones. In our Pavlovian conditioning task, we found no sex differences in cue-evoked dopamine release. When examining reward-evoked dopamine release, both males and females exhibited greater dopamine release following the delivery of the large reward, consistent with prior findings (Tobler et al., 2005; Roesch et al., 2009; Gan et al., 2010). However there were sex differences in the dynamics of the post-reward dopamine response, with lower dopamine levels in females during the first training session. Because we did not monitor the stages of the estrous cycle, we cannot determine if these transient sex differences in reward-evoked dopamine release arise from cycling 
hormones or instead are due to intrinsic differences between males and females. Future studies are needed to determine whether these sex differences with in vivo dopamine transmission are mediated at the level of the cell bodies in the ventral tegmental area or at the dopamine terminals in the NAc.

The magnitude of the dopamine response to the cue presentation and reward delivery have been linked to behavioral outcomes in a variety of Pavlovian conditioning tasks (Darvas et al., 2014; Fonzi et al., 2017; Coddington and Dudman, 2018; Heymann et al., 2020; Lee et al., 2020; Morrens et al., 2020; Stelly et al., 2020; Stelly et al., 2021). We find that conditioned responding correlated with cue-evoked dopamine release in both males and females. Following the large reward delivery, the dopamine response correlated with the number of head entries only in female rats. Further research is necessary to determine if there is a relationship between these post-reward dopamine responses and the post-reward head entries.

Our study identified sex differences in behavior that are sustained over training sessions (conditioned responding, latency, post-reward head entries) as well as transient sex differences in reward-evoked dopamine release. The sex differences in cue-elicited head entries in our Pavlovian task contrasts with a previous Pavlovian study that employed a different cue (compound lever extension / cue light) and reward (liquid sucrose) than what we used in our study (Stringfield et al., 2019). As such, one must be cautious about inferring the presence or absence of sex differences based upon related studies, which underscores the importance of including both males and females in the experimental design. 


\section{References:}

Bakdash JZ, Marusich LR (2017) Repeated Measures Correlation. Front Psychol 8:456.

Becker JB, Chartoff E (2019) Sex differences in neural mechanisms mediating reward and addiction. Neuropsychopharmacology 44:166-183.

Becker JB, Perry AN, Westenbroek C (2012) Sex differences in the neural mechanisms mediating addiction: a new synthesis and hypothesis. Biol Sex Differ 3:14.

Brundage JN, Mason CP, Wadsworth HA, Finuf CS, Nelson JJ, Ronstrom PJW, Jones SR, Siciliano CA, Steffensen SC, Yorgason JT (2021) Regional and sex differences in spontaneous striatal dopamine transmission. J Neurochem.

Calipari ES, Juarez B, Morel C, Walker DM, Cahill ME, Ribeiro E, Roman-Ortiz C, Ramakrishnan C, Deisseroth K, Han MH, Nestler EJ (2017) Dopaminergic dynamics underlying sex-specific cocaine reward. Nat Commun 8:13877.

Castner SA, Xiao L, Becker JB (1993) Sex differences in striatal dopamine: in vivo microdialysis and behavioral studies. Brain Res 610:127-134.

Chen CS, Ebitz RB, Bindas SR, Redish AD, Hayden BY, Grissom NM (2021) Divergent Strategies for Learning in Males and Females. Curr Biol 31:39-50 e34.

Clark JJ, Sandberg SG, Wanat MJ, Gan JO, Horne EA, Hart AS, Akers CA, Parker JG, Willuhn I, Martinez V, Evans SB, Stella N, Phillips PE (2010) Chronic microsensors for longitudinal, subsecond dopamine detection in behaving animals. Nat Methods 7:126-129.

Coddington LT, Dudman JT (2018) The timing of action determines reward prediction signals in identified midbrain dopamine neurons. Nat Neurosci 21:1563-1573.

Darvas M, Wunsch AM, Gibbs JT, Palmiter RD (2014) Dopamine dependency for acquisition and performance of Pavlovian conditioned response. Proc Natl Acad Sci U S A 111:2764-2769.

Dubroqua S, Boison D, Feldon J, Mohler H, Yee BK (2011) Examining the sex- and circadian dependency of a learning phenotype in mice with glycine transporter 1 deletion in two Pavlovian conditioning paradigms. Neurobiol Learn Mem 96:218-229.

Eubig PA, Noe TE, Floresco SB, Sable JJ, Schantz SL (2014) Sex differences in response to amphetamine in adult Long-Evans rats performing a delay-discounting task. Pharmacol Biochem Behav 118:1-9.

Fiorillo CD, Tobler PN, Schultz W (2003) Discrete coding of reward probability and uncertainty by dopamine neurons. Science 299:1898-1902.

Fonzi KM, Lefner MJ, Phillips PEM, Wanat MJ (2017) Dopamine Encodes Retrospective Temporal Information in a Context-Independent Manner. Cell Rep 20:1765-1774.

Gan JO, Walton ME, Phillips PE (2010) Dissociable cost and benefit encoding of future rewards by mesolimbic dopamine. Nat Neurosci 13:25-27.

George BE, Barth SH, Kuiper LB, Holleran KM, Lacy RT, Raab-Graham KF, Jones SR (2021) Enhanced heroin self-administration and distinct dopamine adaptations in female rats. Neuropsychopharmacology. 
Hart AS, Clark JJ, Phillips PEM (2015) Dynamic shaping of dopamine signals during probabilistic Pavlovian conditioning. Neurobiol Learn Mem 117:84-92.

Heien ML, Khan AS, Ariansen JL, Cheer JF, Phillips PE, Wassum KM, Wightman RM (2005) Real-time measurement of dopamine fluctuations after cocaine in the brain of behaving rats. Proc Natl Acad Sci U S A 102:10023-10028.

Heymann G, Jo YS, Reichard KL, McFarland N, Chavkin C, Palmiter RD, Soden ME, Zweifel LS (2020) Synergy of Distinct Dopamine Projection Populations in Behavioral Reinforcement. Neuron 105:909-920 e905.

Kutlu MG, Zachry JE, Brady LJ, Melugin PR, Kelly SJ, Sanders C, Tat J, Johnson AR, Thibeault K, Lopez AJ, Siciliano CA, Calipari ES (2020) A novel multidimensional reinforcement task in mice elucidates sex-specific behavioral strategies. Neuropsychopharmacology 45:1463-1472.

Lee K, Claar LD, Hachisuka A, Bakhurin KI, Nguyen J, Trott JM, Gill JL, Masmanidis SC (2020) Temporally restricted dopaminergic control of reward-conditioned movements. Nat Neurosci 23:209-216.

Lynch WJ, Carroll ME (1999) Sex differences in the acquisition of intravenously selfadministered cocaine and heroin in rats. Psychopharmacology (Berl) 144:77-82.

McArthur S, McHale E, Gillies GE (2007) The size and distribution of midbrain dopaminergic populations are permanently altered by perinatal glucocorticoid exposure in a sexregion- and time-specific manner. Neuropsychopharmacology 32:1462-1476.

Morrens J, Aydin C, Janse van Rensburg A, Esquivelzeta Rabell J, Haesler S (2020) Cue-Evoked Dopamine Promotes Conditioned Responding during Learning. Neuron 106:142-153 e147.

Oliva I, Wanat MJ (2019) Operant Costs Modulate Dopamine Release to Self-Administered Cocaine. J Neurosci 39:1249-1260.

Oliva I, Donate MM, Lefner MJ, Wanat MJ (2021) Cocaine experience abolishes the motivation suppressing effect of CRF in the ventral midbrain. Addict Biol 26:e12837.

Phillips PE, Walton ME, Jhou TC (2007) Calculating utility: preclinical evidence for cost-benefit analysis by mesolimbic dopamine. Psychopharmacology (Berl) 191:483-495.

Pitchers KK, Flagel SB, O'Donnell EG, Woods LC, Sarter M, Robinson TE (2015) Individual variation in the propensity to attribute incentive salience to a food cue: influence of sex. Behav Brain Res 278:462-469.

Roesch MR, Calu DJ, Schoenbaum G (2007) Dopamine neurons encode the better option in rats deciding between differently delayed or sized rewards. Nat Neurosci 10:1615-1624.

Roesch MR, Singh T, Brown PL, Mullins SE, Schoenbaum G (2009) Ventral striatal neurons encode the value of the chosen action in rats deciding between differently delayed or sized rewards. J Neurosci 29:13365-13376.

Salamone JD, Correa M (2012) The mysterious motivational functions of mesolimbic dopamine. Neuron 76:470-485.

Stelly CE, Tritley SC, Rafati Y, Wanat MJ (2020) Acute Stress Enhances Associative Learning via Dopamine Signaling in the Ventral Lateral Striatum. J Neurosci 40:4391-4400. 
Stelly CE, Girven KS, Lefner MJ, Fonzi KM, Wanat MJ (2021) Dopamine release and its control over early Pavlovian learning differs between the NAc core and medial NAc shell. Neuropsychopharmacology.

Stelly CE, Haug GC, Fonzi KM, Garcia MA, Tritley SC, Magnon AP, Ramos MAP, Wanat MJ (2019) Pattern of dopamine signaling during aversive events predicts active avoidance learning. Proc Natl Acad Sci U S A 116:13641-13650.

Stringfield SJ, Madayag AC, Boettiger CA, Robinson DL (2019) Sex differences in nicotineenhanced Pavlovian conditioned approach in rats. Biol Sex Differ 10:37.

Tobler PN, Fiorillo CD, Schultz W (2005) Adaptive coding of reward value by dopamine neurons. Science 307:1642-1645.

Walker QD, Rooney MB, Wightman RM, Kuhn CM (2000) Dopamine release and uptake are greater in female than male rat striatum as measured by fast cyclic voltammetry. Neuroscience 95:1061-1070.

Xiao L, Becker JB (1994) Quantitative microdialysis determination of extracellular striatal dopamine concentration in male and female rats: effects of estrous cycle and gonadectomy. Neurosci Lett 180:155-158.

Yoest KE, Cummings JA, Becker JB (2019) Oestradiol influences on dopamine release from the nucleus accumbens shell: sex differences and the role of selective oestradiol receptor subtypes. Br J Pharmacol 176:4136-4148.

Zachry JE, Johnson AR, Calipari ES (2019) Sex Differences in Value-Based Decision Making Underlie Substance Use Disorders in Females. Alcohol Alcohol 54:339-341.

Zachry JE, Nolan SO, Brady LJ, Kelly SJ, Siciliano CA, Calipari ES (2021) Sex differences in dopamine release regulation in the striatum. Neuropsychopharmacology 46:491-499.

Zhang D, Yang S, Yang C, Jin G, Zhen X (2008) Estrogen regulates responses of dopamine neurons in the ventral tegmental area to cocaine. Psychopharmacology (Berl) 199:625-635. 